\title{
Central Role of High Density Lipoprotein in Plasma
}

\section{Free Cholesterol Metabolism}

\author{
Charles C. Schwartz, Z. Reno Vlahcevic, Mones Berman, John G. Meadows, \\ Richard M. Nisman, and LeON Swell, Division of Gastroenterology, \\ Department of Medicine, Medical College of Virginia, Lipid Research \\ Laboratory, Veterans Administration Medical Center, Richmond, Virginia \\ 23298; Laboratory of Theoretical Biology, National Cancer Institute, \\ National Institutes of Health, Bethesda, Maryland 20014
}

A B S T R A C T This study was designed to provide direct information on the in vivo metabolism in man of free (unesterified) cholesterol in the major lipoprotein classes. Five human subjects were administered one or two (simultaneous) of the following; $\left[2-{ }^{14} \mathrm{C}\right]$ mevalonic acid, high density lipoprotein (HDL)-free $\left[{ }^{14} \mathrm{C}\right]$ cholesterol, low density lipoprotein (LDL)-free $\left[{ }^{14} \mathrm{C}\right]$ cholesterol, and very low density lipoprotein (VLDL)free $\left[{ }^{3} \mathrm{H}\right]$ cholesterol. Blood was then obtained at frequent intervals for at least $9 \mathrm{~h}$, and the $\alpha(\mathrm{HDL})$ and $\beta(L D L+V L D L)$ lipoproteins were quickly separated by heparin-manganese precipitation to prevent ex vivo exchange of free cholesterol. After the administration of $\left[{ }^{14} \mathrm{C}\right]$ mevalonic acid the specific activity (disintegrations per minute/micromole) of free cholesterol in the $\alpha$ - and $\beta$-lipoproteins increased for $3 \mathrm{~h}$. During this period the $\alpha$-free cholesterol specific activity was higher than the $\beta$ specific activity. After administration of VLDL and LDL labeled with free cholesterol, the $\alpha$-free cholesterol specific activity reached a peak value within $20 \mathrm{~min}$, at which time it was considerably lower than the $\beta$-free cholesterol specific activity. When HDL labeled with free cholesterol was administered, a precursor product relationship was observed between the $\alpha$-free cholesterol (precursor) and $\beta$-free cholesterol (product) specific activities.

A multicompartmental model was developed that contained the simplest structure necessary to fit all of the data obtained. The kinetic analysis revealed the presence of extensive exchange $(20-85 \mu \mathrm{mol} / \mathrm{min})$ of free cholesterol between HDL and a tissue pool(s) enriched with newly synthesized free cholesterol. It was found that virtually all ( $>95 \%$ ) of the free cholesterol

Received for publication 14 September 1981 and in revised form 5 February 1982.

Dr. Nisman is an Associate Investigator, Veterans Administration Medical Center. in the $\beta$-lipoproteins (LDL+VLDL) cycles directly through HDL. The free cholesterol in LDL appears to behave in the same fashion as the free cholesterol in VLDL.

The results show that there are marked differences in the kinetic behavior of the free cholesterol fractions of $\alpha$ - and $\beta$-lipoproteins. There is extensive recycling of free cholesterol between HDL and tissue pools, and between HDL and the $\beta$-lipoproteins; this recycling has been quantitated. The findings support the view that in vivo, the free cholesterol in HDL plays a central role in exchange reactions and in the vascular-tissue cholesterol transport system.

\section{INTRODUCTION}

It has been customary to consider lipoprotein free (unesterified) cholesterol as one homogeneous plasma compartment both kinetically and metabolically because of the rapid exchange of free cholesterol observed between the lipoprotein classes. When labeled cholesterol is added to plasma or administered in vivo to rat or man and the lipoproteins are separated, it has been consistently observed that the specific activities of the free cholesterol fraction of the very low density lipoprotein (VLDL), ${ }^{1}$ low density lipoprotein (LDL),

\footnotetext{
${ }^{1}$ Abbreviations used in this paper: $\alpha$, Lipoproteins in the supernate after treatment of plasma with heparin-manganese (considered identical to HDL in this paper); $\beta$, lipoproteins in the precipitate following treatment of plasma with heparin-manganese; $C(n)$, compartment $n$ in the model; DBED, dibenzylethylenediamine; FC, free (unesterified) cholesterol; HDL, high density lipoprotein, $d=1.063-1.21$; $L(n, n)$, fraction of cholesterol in compartment $n$ which leaves compartment $n$ per minute; $L(m, n)$, fraction of cholesterol in compartment $\mathbf{n}$ which is transported to compartment $\mathrm{m}$ per minute; LCAT, lecithin-cholesterol acyltransferase (E.C. 2.3.1.43); LDL, low density lipoprotein, $d=1.019-1.063 ; M(n)$, steady-state mass of cholesterol in
} 
and high density lipoprotein (HDL) are virtually identical (1). Similar observations have been made in man after the administration of labeled mevalonic acid (2). However, it should be pointed out that the observations made in these investigations were based primarily on the isolation of lipoproteins by ultracentrifugation, which takes several days and also on plasma specimens obtained several hours to days after the administration of labeled compounds. However, when the ex vivo exchange of cholesterol was prevented by rapidly separating the lipoproteins and when the plasma was sampled at early time intervals after labeled mevalonic acid administration, the $\alpha$-lipoprotein free cholesterol was shown to have a higher specific activity than the $\beta$-lipoprotein free cholesterol for several hours in the baboon (3). There is also indirect evidence suggesting that lipoprotein free cholesterol is not metabolically homogeneous. An important function of lipoprotein free cholesterol related to the catabolic degradation of cholesterol in man has recently been reported. Multicompartmental kinetic analysis (4) of cholesterol metabolism in man has shown that lipoprotein free cholesterol is the major precursor substrate for bile acid synthesis and biliary cholesterol. Further examination of the metabolism of the lipoprotein free cholesterol fractions indicated that HDL-free cholesterol was preferred over LDL-free cholesterol for bile acid synthesis and biliary cholesterol secretion (5-7). In addition, the free cholesterol in HDL, but not in VLDL or LDL, is used as a substrate for the lecithin-cholesterol acyltransferase (E.C. 2.3.1.43) (LCAT) enzyme $(3,8)$.

HDL cholesterol is of great interest as a coronary risk factor. The plasma HDL cholesterol concentration has been shown to correlate inversely with the incidence of coronary artery disease (9). However, the mechanism by which HDL offers protection is not known. If HDL-free cholesterol is metabolized differently than VLDL- and LDL-free cholesterol, i.e., if heterogeneity exists in the plasma free cholesterol, the protective effect of HDL might potentially be determined.

In the present communication, direct evidence is presented in man to show that plasma free cholesterol is heterogeneous. Subjects were administered isotopic free cholesterol in HDL, LDL, or VLDL, and $\left[{ }^{14} \mathrm{C}\right]$ mevalonic acid. There were large differences be-

C(n) in micromoles; MVA, mevalonic acid; NSC, newly synthesized cholesterol; $R(m, n)$, micromoles of free cholesterol transported from compartment $n$ to compartment $m$ per minute; Turnover time, $1 \div L(n, n)$, the time required for that quantity which is equal to the pool size, $M(n)$, to move out of $C(n) ; U_{n}$, rate of entry of newly synthesized cholesterol into $\mathrm{C}(\mathrm{n})$ in micromoles per minute; VLDL, very low density lipoprotein, $d \leq 1.006$. tween the specific activities of the rapidly isolated $\beta$-lipoproteins (VLDL + LDL) and the $\alpha$, which persisted for $3 \mathrm{~h}$ after the administration of labels. Multicompartmental analysis (SAAM-27) of the data was carried out.

\section{METHODS}

Labeled materials. The dibenzylethylenediamine (DBED) salt of DL $\left[2-{ }^{14} \mathrm{C}\right]$ mevalonic acid was obtained from New England Nuclear, Boston, MA. The mevalonic acid was liberated from the salt by the addition of $7.5 \%$ sodium bicarbonate followed by extraction of DBED with diethylether. The aqueous solution of $\left[2-{ }^{14} \mathrm{C}\right]$ sodium mevalonate was neutralized with an equimolar amount of HCL and diluted to 25 $\mathrm{ml}$ with sterile saline. The solution was passed through a 0.22-micron Millipore filter (Millipore Corp., Bedford, MA) and administered to the subject.

The $\left[4-{ }^{14} \mathrm{C}\right]$ cholesterol and $\left[1,2-{ }^{3} \mathrm{H}\right]$ cholesterol were obtained from New England Nuclear and stored at $-15^{\circ} \mathrm{C}$ in ethanol. The radiopurity $(>95 \%)$ of the labeled cholesterol was checked by recovery of ${ }^{3} \mathrm{H}$ and ${ }^{14} \mathrm{C}$ activity as the digitonide (10), by silicic acid column chromatography (11), and by thin-layer chromatography on silica gel $G$ with a solvent system of petroleum ether/diethyl ether/acetic acid $(89: 11: 3 \mathrm{vol} / \mathrm{vol} / \mathrm{vol})$

Preparation of each labeled lipoprotein was carried out from a fresh fasting sample of the subject's blood. Five 5$\mathrm{mm}$ strips of Whatman No. 1 filter paper (Whatman Inc., Paper Div., Clifton, NJ) were impregnated with [4$\left.{ }^{14} \mathrm{C}\right]$ cholesterol or $\left[1,2-{ }^{3} \mathrm{H}\right]$ cholesterol, and the ethanol was removed under $\mathbf{N}_{2}$. To the paper strips in an incubation flask was added $40 \mathrm{ml}$ of the subject's plasma and the mixture was incubated with agitation at $4^{\circ} \mathrm{C}$ for 90 min under $\mathrm{N}_{2}$. HDL labeled with free $\left[{ }^{14} \mathrm{C}\right]$ cholesterol was then isolated from the incubation mixture as described (12). Briefly, the filter paper strips were removed and the $\beta$-lipoproteins were precipitated from the plasma with heparin $\mathrm{MnCl}_{2}$ (13). The supernatant solution containing the $\alpha$-lipoproteins was dialyzed at $4^{\circ} \mathrm{C}$ overnight against a $5 \%$ solution of $\mathrm{BaCl}_{2}, \mathrm{pH}$ 7.7; the precipitated barium-heparin complex was removed by centrifugation. To the solution was added $7.5 \%$ sodium bicarbonate to precipitate barium and the resultant $\mathrm{BaCO}_{3}$ was removed by centrifugation. The $\alpha$-lipoprotein solution was adjusted to a density of $1.210 \mathrm{~g} / \mathrm{ml}$ with solid $\mathrm{KBr}$, and the solution was centrifuged for $23 \mathrm{~h}$ at $38,000 \mathrm{rpm}$ in a 40 Ti rotor to obtain the HDL fraction.

LDL labeled with free $\left[{ }^{14} \mathrm{C}\right]$ cholesterol was separated from ${ }^{14} \mathrm{C}$-labeled whole plasma by successive ultracentrifugation at $d=1.019$ and $1.063 \mathrm{~g} / \mathrm{ml}$. VLDL labeled with free $\left[{ }^{3} \mathrm{H}\right.$ ]cholesterol was isolated from the top of ${ }^{3} \mathrm{H}$-labeled plasma after ultracentrifugation at $d=1.006 \mathrm{~g} / \mathrm{ml}$. The HDL and LDL preparations were dialyzed overnight against saline containing $0.01 \%$ EDTA, pH 7.4 to remove $\mathrm{KBr}$. All the labeled autologous lipoproteins were passed through 0.45 - and 0.22 -micron Millipore filters immediately before administration to the subjects.

The labeled lipoproteins were checked for homogeneity of mass and label as follows. Over $90 \%$ of the radioactivity migrated with the appropriate lipoprotein zone on agarose gel electrophoresis (14). Upon reultracentrifugation of the labeled lipoproteins, 94-96\% of the ${ }^{14} \mathrm{C}$ and ${ }^{3} \mathrm{H}$ activity was recovered in the appropriate density range. Virtually all the radioactivity was recovered as ${ }^{14} \mathrm{C}$ - and ${ }^{3} \mathrm{H}$-free cholesterol; 
$<0.5 \%$ of the ${ }^{3} \mathrm{H}$ or ${ }^{14} \mathrm{C}$ was in the esterified cholesterol fraction; preparation at $4^{\circ} \mathrm{C}$ prevented LCAT activity.

Subjects. The experiments were carried out on five subjects from whom informed consent was obtained. They all had normal renal and liver function tests, normal lipoprotein profiles, no evidence of diabetes mellitus, and were in a good state of nutrition. Pertinent information on the subjects is shown in Table I. The five subjects were fasted overnight. The labeled compounds shown in Table I were administered intravenously at 10 a.m. over a 2 -min period. Blood samples were then obtained at frequent intervals (as indicated in Figs. 1-5) for the first $9 \mathrm{~h}$ and then less frequently for the next several days.

Lipoprotein cholesterol analysis. Blood samples were collected in heparinized (143 USP units) tubes and were then immediately centrifuged at $4^{\circ} \mathrm{C}$ for $5 \mathrm{~min}$ to separate the plasma from erythrocytes. The plasma was then immediately treated with heparin- $\mathrm{MnCl}_{2}$ (13) to precipitate the $\beta$-lipoproteins, which were then removed by centrifugation, washed, and reprecipitated; the $\alpha$-lipoproteins remained in the original supernatant. The identity of the $\alpha$ - and $\beta$-lipoprotein fractions were verified by agarose gel electrophoresis (14). The $\alpha$-lipoprotein fraction corresponded to HDL $(d=1.063 \leq 1.21)$ and was free of $\beta$-lipoproteins. The $\beta$ fraction corresponded to VLDL plus LDL $(d<1.063)$ and did not contain $\alpha$-lipoproteins. Both the $\alpha$ - and the $\beta$-fractions were extracted with $20 \mathrm{vol}$ of $2: 1$ chloroform/methanol. After the addition of one-fifth vol of water (15), the lower chloroform phase was collected and subjected to silicic acid column chromatography (11) to separate the free cholesterol from esterified cholesterol.

Lipoprotein-free cholesterol mass was determined on an aliquot of the free cholesterol column fraction by gas liquid chromatography using coprostanol as the internal standard. The stationary phase was 3\% SP 2401 (Supelco, Inc., Bellefonte, PA). Carrier free cholesterol was added to another aliquot of the free cholesterol fraction, the digitonide formed, isolated, dissolved in methanol, and ${ }^{14} \mathrm{C}$ or ${ }^{3} \mathrm{H}$ activity was determined by liquid scintillation counting in Liquifluor (New England Nuclear). The isolated digitonides were checked for the presence of labeled contaminants by cleaving the digitonide with pyridine and determining the distribution of radioactivity on TLC. Virtually all the (>99\%) radioactivity was recovered in the free cholesterol zone.

Radioactivity was determined in a Mark III Liquid Scintillation Counter (Tracor Analytic Inc., Silver Springs, MD); quench correction was applied using the external standard method.

\section{RESULTS}

Lipoprotein cholesterol concentrations. The plasma concentrations of $\alpha$ and $\beta$ free cholesterol for the five subjects during the experiments are shown in Table I. There was a wide range of $\alpha$-FC concentration from subject to subject $(15-43 \mu \mathrm{mol} / \mathrm{dl})$. However, within each subject the $\alpha$-FC concentration was constant during the course of the experiment as denoted by the small SEM. Also, no trends in the concentrations were noted during each experiment. The $\beta$-FC concentrations varied less from subject to subject and were also constant within each subject. No trends were noted in the $\beta$-FC levels. The constancy of the plasma lipopro- tein free cholesterol and esterified cholesterol concentrations are suggestive of a steady state of cholesterol metabolism during the $9 \mathrm{~h}$ to $3 \mathrm{~d}$ of the experiments.

Specific activity data. Two factors were found to be critical in demonstrating differences in the kinetic behavior of the free cholesterol of the $\alpha$ - and $\beta$-lipoproteins. The first was the rapid separation of the lipoproteins to minimize ex vivo cholesterol exchange after the blood specimen was taken from the subject and second was frequent sampling of blood during the first few hours after administration of labeled preparations. Blood samples were obtained from subjects 2 , 4 , and 5 , at intervals during the initial $3 \mathrm{~h}$ after administration of labels. One aliquot of each blood sample was subjected to ultracentrifugal isolation of $\mathrm{HDL}$, LDL, and VLDL, and from another aliquot the $\alpha$ - and $\beta$-lipoproteins were quickly separated. The specific activity of free cholesterol (FC) in the lipoproteins isolated by the two methods are shown for comparison in Table II. Within each ultracentrifugal plasma sample no consistent differences were noted in the specific activities of the FC of HDL, VLDL, and LDL, whereas systematic differences did exist between the specific activities of the $\alpha$ - and $\beta$-lipoproteins.

The specific activity vs. time curves of the free cholesterol in $\alpha$ - and $\beta$-lipoproteins for subjects 1,2 , and 3 are shown in Figs. 1-3. These subjects received either one or two lipoprotein preparations containing free $\left[{ }^{3} \mathrm{H}\right]$ and/or $\left[{ }^{14} \mathrm{C}\right]$ cholesterol.

Subject 1 received VLDL labeled with free $\left[{ }^{3} \mathrm{H}\right]$ cholesterol (Fig. 1, top). The ${ }^{3} \mathrm{H}-\beta-F C$ specific activity decreased in a multiexponential fashion. The ${ }^{3} \mathrm{H}$ $\alpha$-FC specific activity increased rapidly reaching a maximum (peak) after $\sim 20 \mathrm{~min}$, and then decreased slowly to merge with the ${ }^{3} \mathrm{H}-\beta-\mathrm{FC}$ after $\sim 180 \mathrm{~min}$. Most notable was the observation that the ${ }^{3} \mathrm{H}-\alpha-\mathrm{FC}$ peak specific activity value was considerably lower than the ${ }^{3} \mathrm{H}-\beta$-FC specific activity at $20 \mathrm{~min}$, and remained lower for $180 \mathrm{~min}$. Subject 3 also received VLDL labeled with free $\left[{ }^{3} \mathrm{H}\right]$ cholesterol (Fig. 2, top) and simultaneously received LDL labeled with free $\left[{ }^{14} \mathrm{C}\right]$ cholesterol (Fig. 2, bottom). For both ${ }^{3} \mathrm{H}$ and ${ }^{14} \mathrm{C}$ data, the relationships observed between the $\alpha$ - and $\beta$-FC specific activity curves were the same as those described above for subject 1 (Fig. 1, top).

During the initial 20-30 min of these two experiments a large portion of the radioactivity in the $\alpha$ lipoprotein fraction is probably acquired directly by transfer from the labeled VLDL or LDL. Potential explanations of the relatively low peak of $\alpha$-FC specific activity include: (a) input of unlabeled free cholesterol to HDL from LDL after ${ }^{3} \mathrm{H}-\mathrm{VLDL}$ administration or from VLDL after ${ }^{14} \mathrm{C}-\mathrm{LDL}$ administration; (b) Input of unlabeled free cholesterol to HDL from tissue (membrane) sources; (c) subcompartments of 


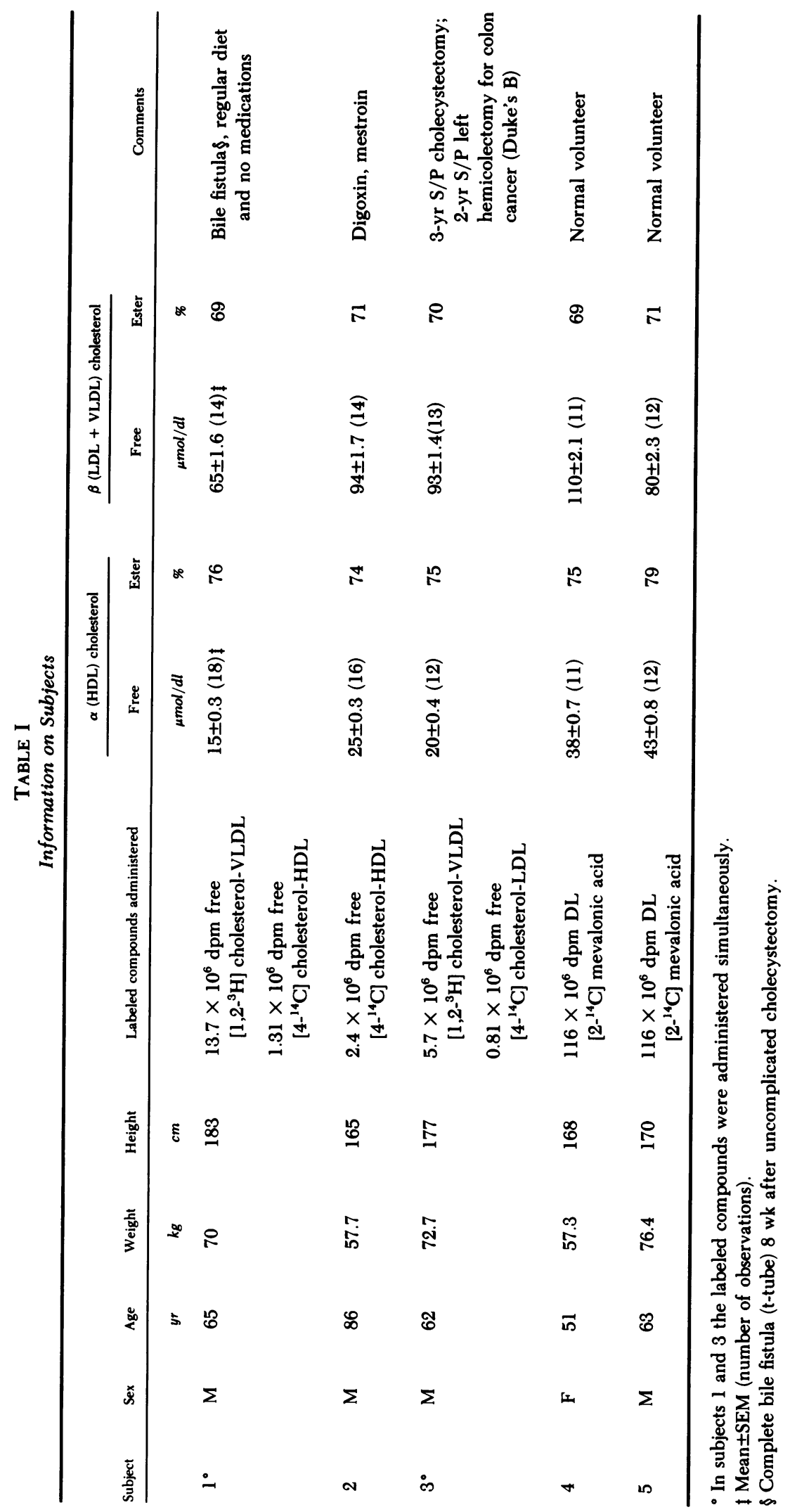


TABLE II

Free Cholesterol Specific Activity in Lipoproteins Isolated from Plasma by Immediate Treatment with Heparin-manganese or by Ultracentrifugation

\begin{tabular}{|c|c|c|c|c|c|c|}
\hline \multirow[b]{2}{*}{ Subject } & \multirow{2}{*}{$\begin{array}{c}\text { Time } \\
\text { after label }\end{array}$} & \multicolumn{5}{|c|}{ Specific activity } \\
\hline & & $\alpha$ & $\beta$ & HDL & LDL $^{\cdot}$ & VLDL \\
\hline & $\min$ & & & $m^{14} \mathrm{C} /$ & & \\
\hline \multirow[t]{6}{*}{$2 \ddagger$} & 9 & 1,297 & 420 & 585 & 561 & 580 \\
\hline & 45 & 472 & 516 & 499 & 402 & 520 \\
\hline & 68 & 334 & 481 & 407 & 379 & 413 \\
\hline & 99 & 321 & 397 & 359 & 372 & 364 \\
\hline & 122 & 262 & 359 & 302 & 299 & 344 \\
\hline & 137 & 260 & 351 & 329 & 305 & 320 \\
\hline \multirow[t]{4}{*}{48} & 20 & 128 & 46 & 60 & 52 & \\
\hline & 30 & 351 & 98 & 114 & 117 & \\
\hline & 65 & 597 & 355 & 406 & 364 & \\
\hline & 180 & 976 & 905 & 895 & 896 & \\
\hline \multirow[t]{4}{*}{$5 \$$} & 20 & 62 & 32 & 36 & 32 & \\
\hline & 30 & 241 & 85 & 115 & 124 & \\
\hline & 60 & 572 & 234 & 275 & 276 & \\
\hline & 180 & 815 & 841 & 739 & 751 & \\
\hline
\end{tabular}

- Represents specific activity of FC in lipoproteins of density 1.0191.063 (LDL) in subject 2, and $d \leq 1.063$ (VLDL, IDL, and LDL) in subjects 4 and 5 .

† Administered HDL free $\left[{ }^{14} \mathrm{C}\right]$ cholesterol.

\& Administered $\left[2-{ }^{14} \mathrm{C}\right]$ mevalonic acid

HDL. The last explanation appears unlikely because of the absence of a slow rise or plateau in the $\alpha$-FC specific activity after the initial rapid incline.

In subject 3, (Fig. 2) who was simultaneously administered VLDL-free $\left[{ }^{3} \mathrm{H}\right]$ cholesterol and LDL-free $\left[{ }^{14} \mathrm{C}\right]$ cholesterol, the specific activities were calculated as fraction of dose per micromole. Expressed in this manner the $\alpha-F C{ }^{14} \mathrm{C}$ and ${ }^{3} \mathrm{H}$ specific activities were essentially identical. This finding could be fortuitous, but is strongly suggestive of similarity of metabolism (homogeneity) of free cholesterol in VLDL and LDL.

Subjects 1 and 2 received HDL labeled with free $\left[{ }^{14} \mathrm{C}\right]$ cholesterol (Fig. 1, bottom; and Fig. 3). In both experiments there was a rapid decline in $\alpha$-FC specific activity during the initial $30 \mathrm{~min}$. This initial decline appeared to be considerably steeper than the initial decline observed in $\beta$-FC after labeled LDL or VLDL administration (Fig. 1, top; and Fig. 2). After $30 \mathrm{~min}$, the rapid decline of $\alpha$-FC specific activity abruptly slowed. The ${ }^{14} \mathrm{C}-\beta$-FC specific activity increased rapidly reaching a maximum in $\sim 25 \mathrm{~min}$. At this time the $\beta$-FC specific activity was similar to that in the $\alpha$-FC fraction; the $\beta$-FC then declined in a fashion compatible with a precursor product relationship-with $\alpha-F C$ as precursor and $\beta-F C$ as product.
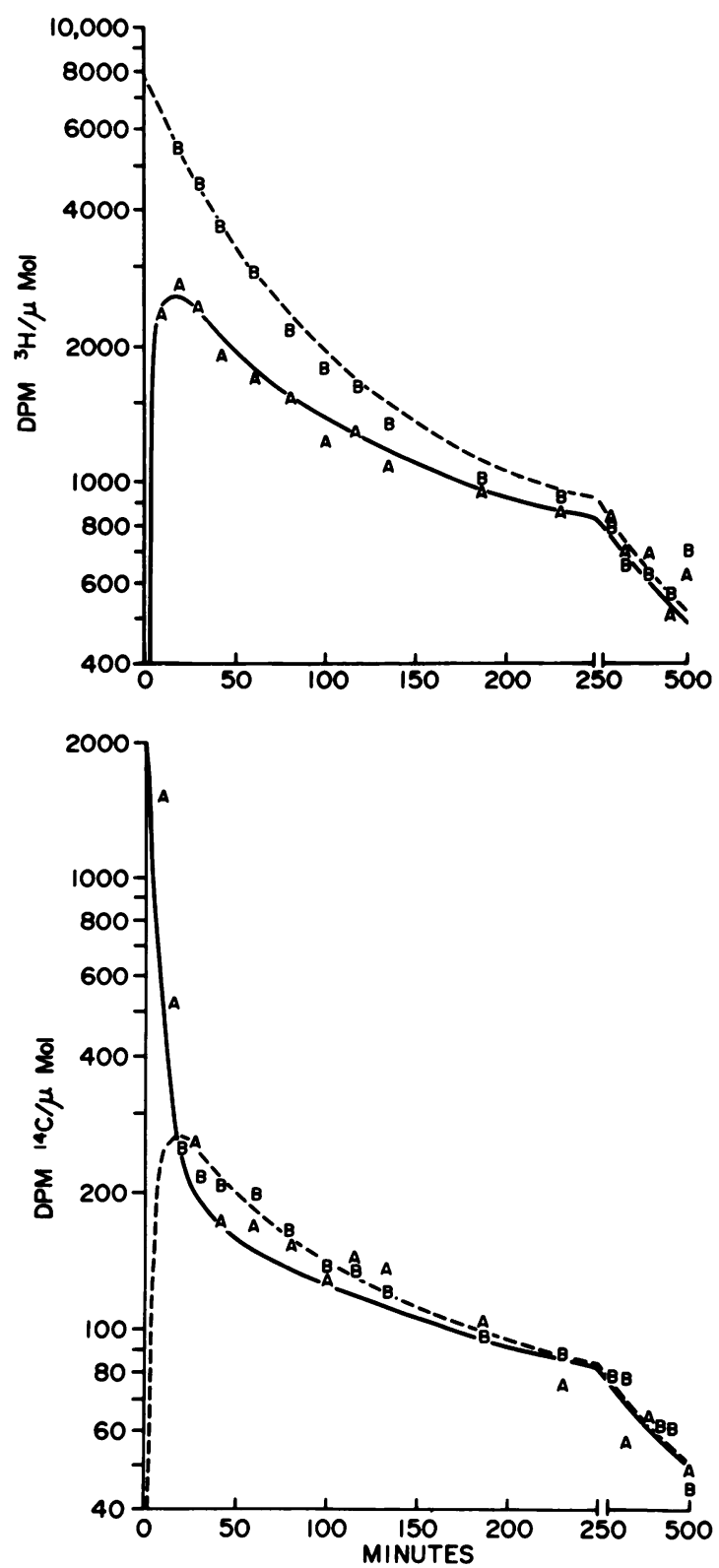

Figure 1 Subject 1 . Observed specific activity values of free cholesterol in $\alpha(A)$ and $\beta(B)$ lipoproteins after simultaneous administration of VLDL-free $\left[{ }^{3} \mathrm{H}\right]$ cholesterol (upper) and HDL-free $\left[{ }^{14} \mathrm{C}\right] \mathrm{cholesterol}$ (lower). The solid and dashed lines represent the computer predictions for the $\alpha-F C$ and $\beta-F C$ values, respectively, using the model in Fig. 6. The ${ }^{3} \mathrm{H}$ and ${ }^{14} \mathrm{C}$ data were simultaneously fit, using identical model parameters.

The free cholesterol specific activity time curves for the two subjects who received $\left[2-{ }^{14} \mathrm{C}\right]$ mevalonic acid are shown in Figs. 4 and 5 . In both subjects the specific activity of the $\alpha$-lipoprotein-free cholesterol fraction rose more rapidly and was higher for the first $180 \mathrm{~min}$ than the $\beta$-lipoprotein fraction. The cross-over of the 

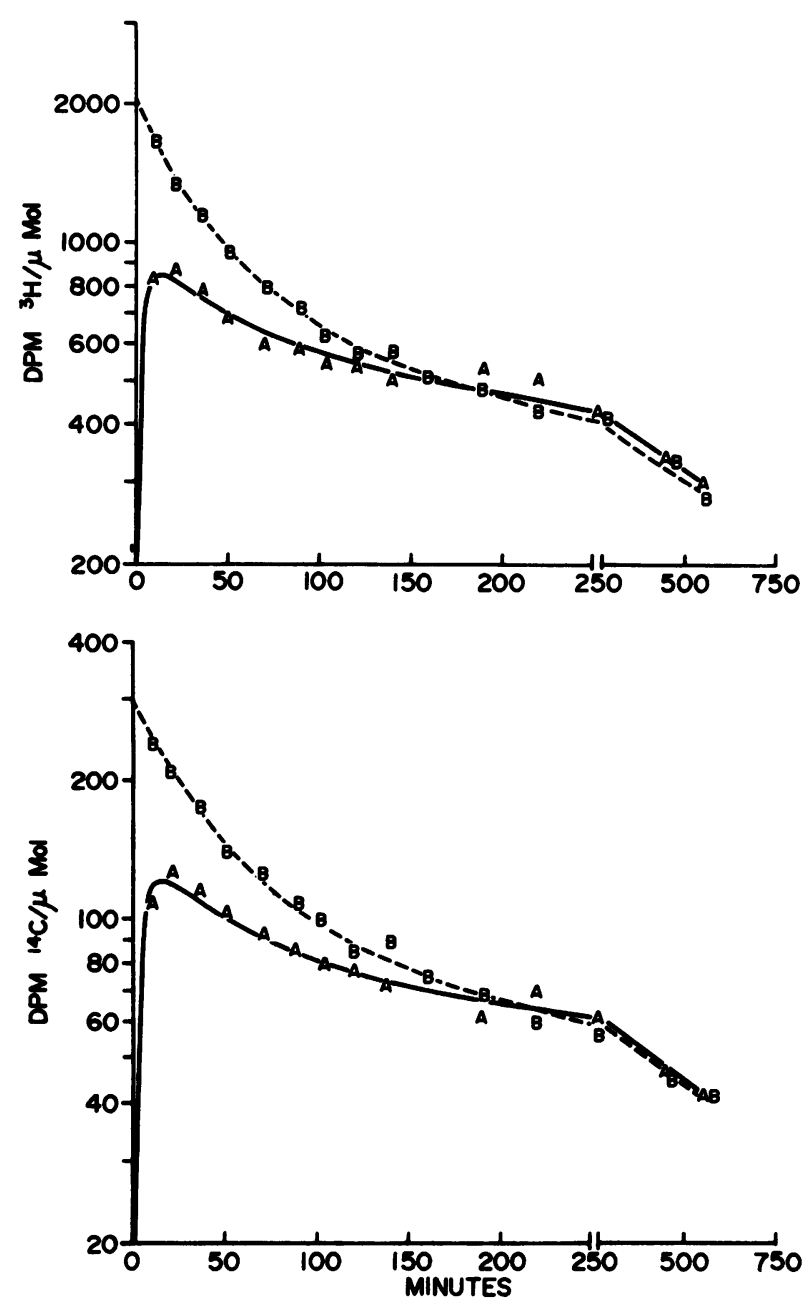

Figure 2 Subject 3. Observed specific activity values of free cholesterol in $\alpha(A)$ and $\beta(B)$ lipoproteins after simultaneous administration of VLDL-free ${ }^{3} \mathrm{H}$ cholesterol (upper) and LDL free $\left[{ }^{14} \mathrm{C}\right]$ cholesterol (lower). The lines represent the computer predictions for the $\alpha-F C$ (solid) and $\beta$-FC (dashed) values when fit to the model in Fig. 6 . The ${ }^{3} \mathrm{H}$ and ${ }^{14} \mathrm{C}$ data were fit simultaneously to the same set of parameters.

$\alpha$ - and $\beta$-lipoprotein specific activity curves occurred at the peak specific activity of the $\beta$-lipoprotein. The higher specific activity of the $\alpha$-lipoprotein fraction initially after $\left[{ }^{14} \mathrm{C}\right]$ mevalonic acid administration clearly indicates that the $\alpha$-lipoprotein fraction was acquiring (directly or indirectly) free cholesterol that had been newly synthesized in the tissues.

Development of the model. The final model arrived at by simulation analysis is shown in Fig. 6. Except for the pathway $R(6,21)$, this model contains the minimum structure necessary to fit the lipoprotein free cholesterol specific activity data obtained after the administration of labeled mevalonic acid, HDL-

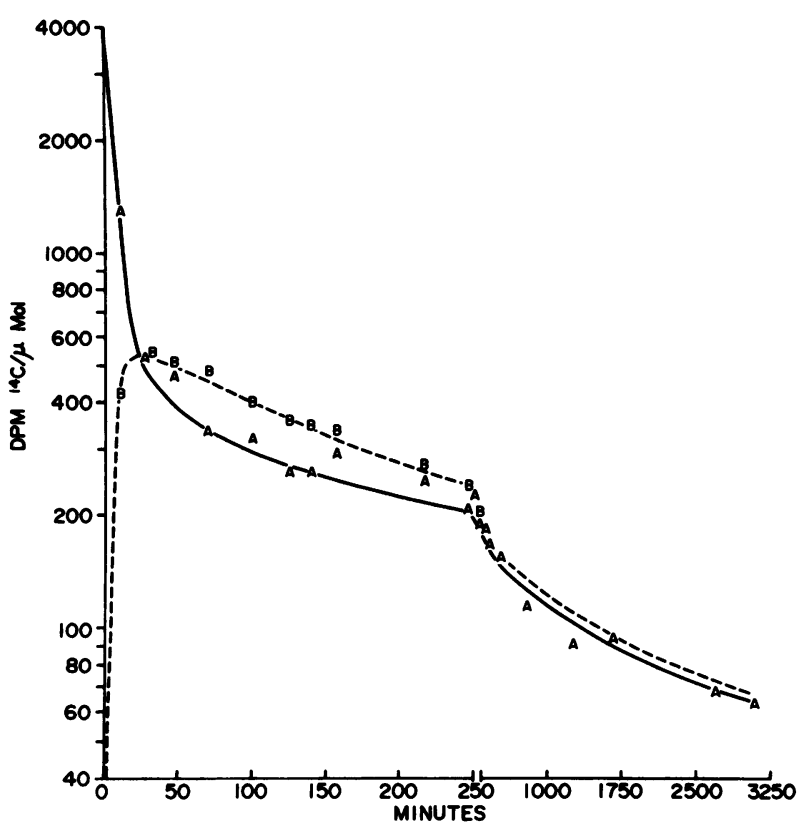

Figure 3 Subject 2. Observed specific activity values of free cholesterol in $\alpha(A)$ and $\beta(B)$ lipoproteins after administration of HDL-free $\left[{ }^{14} \mathrm{C}\right] \mathrm{cholesterol}$. The solid and dashed lines represent the computer predictions for the $\alpha-\mathrm{FC}$ and $\beta-\mathrm{FC}$ values, respectively.

FC, LDL-FC, and VLDL-FC. When ${ }^{3} \mathrm{H}-$ and ${ }^{14} \mathrm{C}-\mathrm{la}-$ beled lipoproteins were administered simultaneously (subjects 1 and 3 ) the ${ }^{3} \mathrm{H}$ and ${ }^{14} \mathrm{C}$ data were simulated and fit simultaneously to the same set of model parameters. The simulation analysis was carried out with the SAAM-27 computer program (16) assuming steadystate kinetics.

Because LDL and VLDL individually labeled with free cholesterol were separately injected (subjects 1 and 3), LDL and VLDL were assumed to comprise separate compartments of free cholesterol in the initial phases of compartmental analysis. The computer predictions for the specific activities of free cholesterol in $L D L, C(21)$, and VLDL, $C(22)$, were added algebraically at each time interval and compared with that actually observed in the $\beta$-FC fraction of plasma, $C(15)$. In the kinetic analysis the mass of $C(15)$ was fixed to that observed, i.e., calculated from $\beta$-FC concentration in Table I and plasma volume as $4.5 \%$ of $\mathrm{kg}$ body weight. The mass of $\beta$-FC $[\mathrm{C}(15)]$ was proportioned $2: 3$ to $C(21)$ and $1: 3$ to $C(22)$, based on previous observations. However, the ratio of LDL to VLDL-FC mass was subsequently found to have no effect on the rate constants $L(4,22), L(4,21)$, or the sum of $L(22,4)$ and $L(21,4)$.

The data from subject 3 who received VLDL ${ }^{3} \mathrm{H}$ FC and $L D L{ }^{14} \mathrm{C}-\mathrm{FC}$ gave the best definition of the 


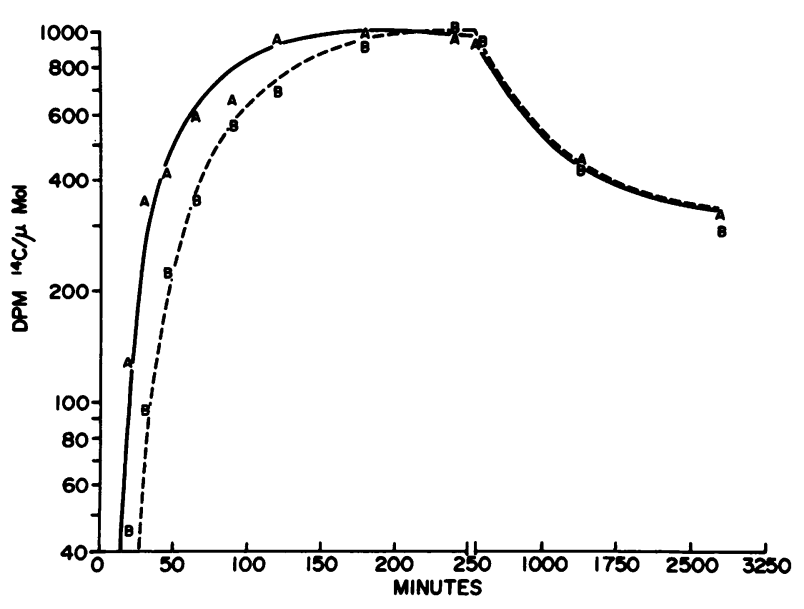

FigURE 4 Subject 4. Observed specific activity values of free cholesterol in $\alpha(A)$ and $\beta(B)$ lipoproteins after administration of $\left[{ }^{14} \mathrm{C}\right]$ mevalonic acid. The solid and dashed lines represent the computer predictions for the $\alpha-F C$ and $\beta-F C$ values, respectively, using the model in Fig. 6.

$\beta$-FC system (VLDL + LDL). This subject's data was simulated under two sets of conditions. First, no exchange of FC occurred between LDL and VLDL as shown in Fig. 6. To simulate the observed $\alpha-F C$ and $\beta$-FC specific activities and pool sizes, the great majority (90-100\%) of the free cholesterol leaving the VLDL and LDL compartments was found to be transported directly to HDL. Also, the rate constants $\mathrm{L}(4$, 22) and $L(4,21)$ were found to be identical $(0.028$ $\left.\min ^{-1}\right)$. The $\beta$-FC fit was optimal with $\alpha$-lipoprotein

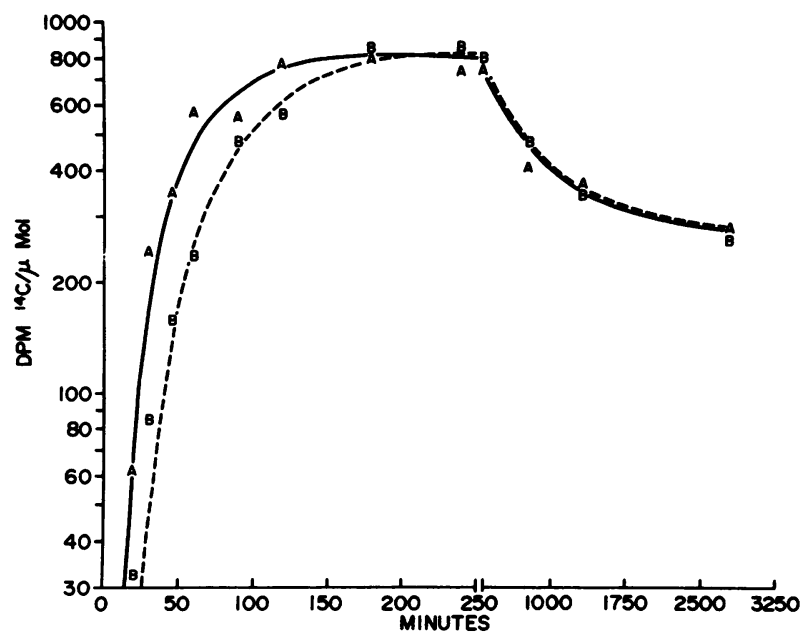

Figure 5 Subject 5 . Observed specific activity values of free cholesterol in $\alpha(A)$ and $\beta(B)$ lipoproteins after administration of $\left[{ }^{14} \mathrm{C}\right]$ mevalonic acid. The lines represent the computer predictions for the $\alpha$-FC (solid) and $\beta$-FC (dashed) values using the model in Fig. 6. as its only source of free cholesterol. Second, the data were simulated assuming various degrees of bidirectional transport between VLDL and LDL. The results obtained were the same as above in that to fit the data (a) almost all (90-100\%) FC leaving the VLDL + LDL fraction was transferred directly to $\mathrm{HDL},(b)$ an optimal fit of the $\beta$-FC was obtained with $\alpha-\mathrm{FC}$ as its only precursor, and (c) the $\beta$-FC fit could not be improved upon when the rate constants $L(4,22)$ and/or $\mathrm{L}(4,21)$ were varied from $0.028 \mathrm{~min}^{-1}$. Because the magnitude of FC transport pathways between VLDL and LDL-FC were not defined by the data and because their presence had no effect on the remaining structure of the model or on the parameters of the $\alpha$-FC and total $\beta$-FC fractions, the modeling was performed without these pathways.

As shown in Fig. 6, a pathway, $R(6,21)$, from $L D L$ FC to a nonplasma compartment, $\mathrm{C}(6)$, was incorporated into the model to represent the process for cellular uptake of LDL FC (17). The rate constant $L(6$, 21) was fixed at a value determined for LDL Apo B from the literature $(18,19)$. Although our study did not allow the detection of $R(6,21)$, its incorporation into the model was found to not effect any part of the solution. The pathway $R(6,21)$ was included in the model because it's existence has been clearly documented by others and for comparison with the magnitude of the new pathways that were defined by the study.

The $\alpha$-HDL-free cholesterol, C(4), was initially modeled as a single compartment as shown in Fig. 6 . Two important observations were made. First, it was found that the initial steep decline in $\alpha$-FC specific activity after HDL-free $\left[{ }^{14} \mathrm{C}\right]$ cholesterol administration (Figs. 1, bottom, and 3 ) could not be attributed solely to transfer of FC from the $\alpha$ - to the $\beta$-fraction. To simulate this steep decline it was necessary to incorporate a pathway from HDL-FC to a nonplasma compartment, designated as $C(12)$. Second, the major pathway of FC transport from nonplasma to plasma compartments was found to be into HDL. This pathway, $L(4,12)$, and not the presence of HDL subcompartments or the input of unlabeled VLDL or LDLFC to HDL, was necessary to simulate the low peak in $\alpha$-FC specific activity shown in Figs. 1 (top) and 2 (top and bottom). A relatively small ( $<15 \%$ of that into HDL) flux of free cholesterol from a nonplasma pool directly into LDL or VLDL could not be excluded, but such an addition did not improve the fit of the data. In summary, bidirectional transport of FC between one HDL pool and a single nonplasma pool, $\mathrm{C}(12)$, resulted in optimal fits of the $\alpha$ - and $\beta$-FC specific activity vs. time curves through the first $9 \mathrm{~h}$ of the experiments. The incorporation of plasma HDL FC subcompartments into the model did not affect 


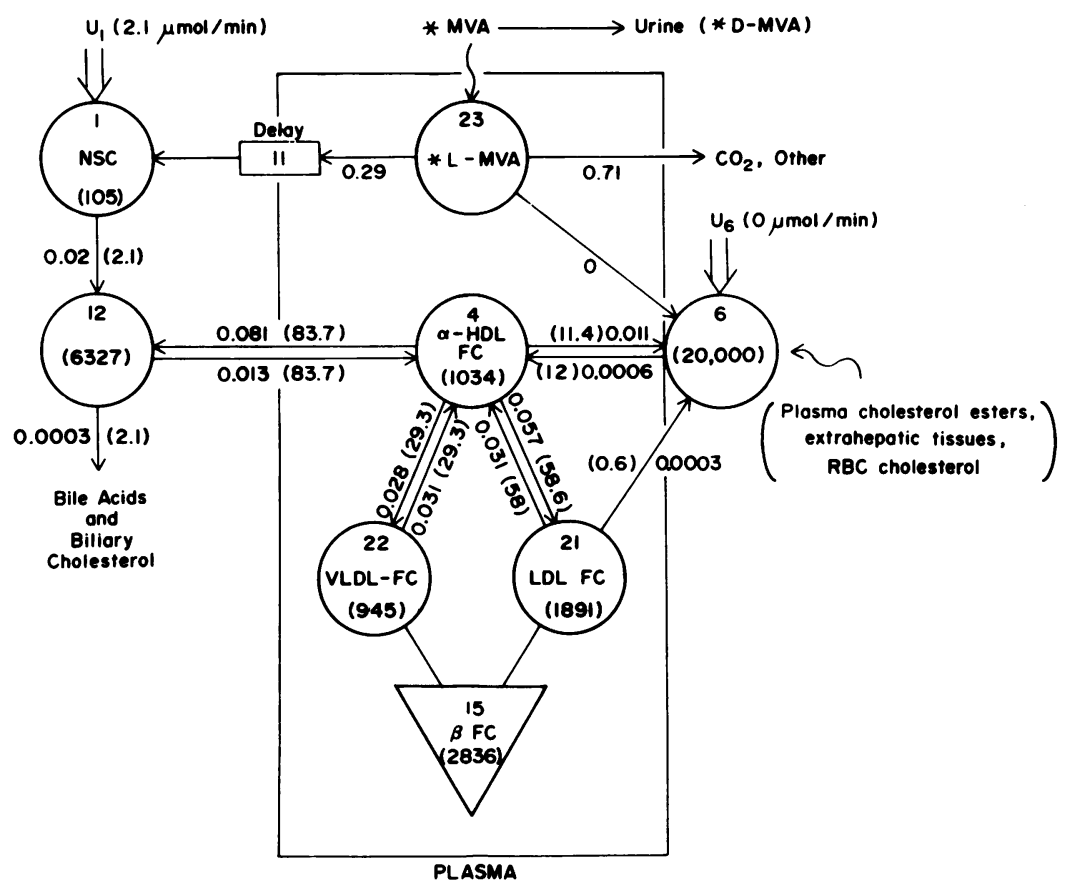

FIGURE 6 The free cholesterol model including parameter values for subject 4 . Rate constants (per minute) are adjacent to arrow; fluxes $(\mu \mathrm{mol} / \mathrm{min})$ are in parentheses adjacent to arrow; pool sizes $(\mu \mathrm{mol})$ are in parentheses inside the compartment. The entire input of cholesterol has been arbitrarily placed into compartment $1\left(U_{6}=0\right) .^{\circ}$, labeled compound administered.

either the necessity for $\mathrm{C}(12)$ or the magnitude of the model parameters.

Subjects 4 and 5 were administered $\left[{ }^{14} \mathrm{C}\right]$ mevalonic acid and the $\alpha$ - and $\beta$-lipoproteins were rapidly separated from blood. The $\alpha$ - and $\beta$-FC specific activities were fit (Figs. 4 and 5) using the model shown in Fig. 6. There was a short delay $(10-11 \mathrm{~min})$ in the incorporation of ${ }^{14} \mathrm{C}$ into free cholesterol accounted for by $\mathrm{C}(11)$. This delay time most likely makes up part of the longer delays, $C(7)$ and $C(10)$, found in previous modeling of the bile acid and biliary cholesterol pools after labeled mevalonic acid (4). The parameter $L(1$, 1) could not be defined by the data and was fixed at $0.020 / \mathrm{min}$ based on the average from previous studies (4).

The need for a relatively slowly exchanging compartment, $C(6)$, appeared in the experiments extended beyond $9 \mathrm{~h}$ (subjects 2,4 , and 5 ). Without $\mathrm{C}(6)$, the simulated specific activities in the $\alpha$ - and $\beta$-FC fractions declined more rapidly after $9 \mathrm{~h}$ than the observed specific activities. The synthesis of cholesterol in this presumed extrahepatic tissue pool could not be determined as it has been in more prolonged experiments (20), and $U_{6}$ has been fixed at zero.

The rate of cholesterol synthesis, $U_{1}$, in subject 1 with a total bile fistula was fixed at $5.5 \mu \mathrm{mol} / \mathrm{min}$, the measured rate of bile acid plus biliary cholesterol excretion. The rate of cholesterol synthesis in subjects 25 was assumed to equal $2.1 \mu \mathrm{mol} / \mathrm{min}(21)$. The specific activity fits and parameter values were not sensitive to this assumption. For example, a twofold change in $U_{1}$ only affected the parameters $L(4,12)$ and $L(12,4)$, and the maximum change was $<10 \%$. The compartment from which cholesterol is lost from the body (bile acid and biliary cholesterol precursor pools) could not be identified precisely from the present experimental data. However, the slow fractional rate constant of $\mathrm{C}(6)$ of $\sim 0.0006 \mathrm{~min}^{-1}$ excludes this pool as the bile acid and/or biliary cholesterol precursor. The latter have rate constants of $\sim 0.015 \mathrm{~min}^{-1}$ (4). Other studies have shown that HDL-FC is excreted at a more rapid rate in bile than LDL (5-7) or VLDLFC (unpublished observations) and also that the bile acid and biliary cholesterol precursor pools are directly supplied, in part, by NSC (4). Because C(12) exchanges FC with HDL and not directly with LDL or VLDL, receives newly synthesized cholesterol (NSC) and has a rate constant of $\sim 0.010 \mathrm{~min}^{-1}$, it could conceivably be comprised in part by the hepatic bile acid and biliary cholesterol precursor pools. Therefore, the output from the free cholesterol model shown in Fig. 6 has been arbitrarily placed at $C(12)$; however, the data 
TABLE III

Steady-state Fluxes Derived from Compartmental Analysis

\begin{tabular}{|c|c|c|c|c|c|c|c|c|}
\hline Subject & $\mathrm{U}(1)^{\bullet}$ & $\mathbf{R}(6,4)$ & $\mathbf{R}(12,4)$ & $\begin{array}{c}\mathbf{R}(22,4) \\
+ \\
\mathbf{R}(21,4)\end{array}$ & $R(4,6)$ & $R(4,12)$ & $\begin{array}{c}\mathbf{R}(4,22) \\
+ \\
\mathbf{R}(4,21)\end{array}$ & $\mathbf{R}(\mathbf{6}, 21)$ \\
\hline & \multicolumn{8}{|c|}{$\mu \mathrm{mol} / \mathrm{min}$} \\
\hline 1 & 5.5 & $12.7 \pm 120 \%$ & $32.9 \pm 41 \%$ & $64.3 \pm 8 \%$ & $13.5 \pm 115 \%$ & $32.9 \pm 39 \%$ & $63.5 \pm 8 \%$ & 0.8 \\
\hline 2 & 2.1 & $4.0 \pm 40 \%$ & $18.5 \pm 26 \%$ & $46.9 \pm 15 \%$ & $4.5 \pm 35 \%$ & $18.5 \pm 27 \%$ & $46.4 \pm 15 \%$ & 0.5 \\
\hline 3 & 2.1 & $11.4 \pm 68 \%$ & $63.0 \pm 9 \%$ & $86.0 \pm 9 \%$ & $11.9 \pm 64 \%$ & $63.0 \pm 8 \%$ & $85.5 \pm 9 \%$ & 0.6 \\
\hline 4 & 2.1 & $11.4 \pm 29 \%$ & $83.7 \pm 37 \%$ & $87.9 \pm 14 \%$ & $12.0 \pm 29 \%$ & $83.7 \pm 38 \%$ & $87.3 \pm 14 \%$ & 0.6 \\
\hline 5 & 2.1 & $13.2 \pm 20 \%$ & $59.6 \pm 34 \%$ & $90.7 \pm 12 \%$ & $13.7 \pm 19 \%$ & $59.6 \pm 34 \%$ & $90.2 \pm 12 \%$ & 0.5 \\
\hline
\end{tabular}

- Estimated from the literature for subjects 2-5 with intact enterohepatic circuits (21); determined experimentally in subject 1 as the sum of bile acid plus biliary cholesterol excretion from the complete bile fistula. In all subjects, $U(1)=R(0,12)=R(12,1)$.

$\downarrow$ Parameter value \pm FSD $($ FSD $=$ SD $\times 100 \div$ parameter value)

shown in Figs. 1-5 were fit equally well with essentially identical solutions when the output was changed from $C(12)$ to $C(6), C(4)$, or $C(15)$.

Model parameters. The fluxes derived from compartmental analysis are shown in Table III. The total free cholesterol flux out of HDL was very large and ranged from 69 to $183 \mu \mathrm{mol} / \mathrm{min}$. Approximately one half of this was transported to the $\beta$-fraction, $R(22,4)$ plus $R(21,4)$. About two-fifths of the total flux from the HDL-FC was into the nonplasma compartments, $C(6)$ and $C(12)$, predominantly via $R(12,4)$. To fit the data, virtually all the $\beta$-FC exchanged only with HDL. In subjects 1 and 3 , in whom the experiments were limited to $9 \mathrm{~h}$, the relatively slow exchanging C(6) was poorly defined as reflected in the large FSD associated with this compartment.

The large amount of free cholesterol that recycles (exchanges) between HDL and the tissue and $\beta$-frac- tions is highlighted by comparison with the flux via the pathway $R(6,21)$. The latter pathway represents the 0.5 to $0.8 \mu \mathrm{mol} / \mathrm{min}$ of LDL-FC, which is internalized by tissues via the uptake of LDL Apo B. Although of large magnitude, the FC exchange pathways between HDL and both $C(12)$ and the $\beta$-fractions are well defined, as reflected by their low FSD.

The rate constants and delay times derived from compartmental analysis are shown in Table IV. All pool sizes derived from the analysis are shown in Table $\mathrm{V}$. The extent of variation in the size of $\mathrm{C}(12)$, the rapidly exchanging tissue pool, was small and ranged from 4,491 to $6,901 \mu \mathrm{mol}$. In most subjects the size of $\mathrm{C}(12)$ was approximately twice the size of the total plasma FC pool. Confidence was gained in the model structure by the finding of similar values between subjects for most of the adjustable rate constants and masses. This similarity is remarkable because of the

TABLE IV

Rate Constants and Delay Times Derived from Compartmental Analysis ${ }^{\circ}$

\begin{tabular}{|c|c|c|c|c|c|c|c|c|c|}
\hline Subject & $L(6,4)$ & $L(12,4)$ & $\begin{array}{c}L(21,4) \\
+ \\
L(22,4)\end{array}$ & $L(4,6)$ & $L(4,12)$ & $L(4,21) !$ & $L(6,21) \S$ & $L(1,23)$ & $t_{11} 1$ \\
\hline & \multicolumn{8}{|c|}{$\min ^{-1}$} & $\min$ \\
\hline 1 & $0.025 \pm 130 \% \pi$ & $0.065 \pm 47 \%$ & $0.127 \pm 23 \%$ & $0.0005 \pm 200 \%$ & $0.007 \pm 59 \%$ & $0.031 \pm 8 \%$ & 0.0006 & & \\
\hline 2 & $0.006 \pm 38 \%$ & $0.029 \pm 22 \%$ & $0.072 \pm 24 \%$ & $0.0003 \pm 47 \%$ & $0.003 \pm 30 \%$ & $0.019 \pm 15 \%$ & 0.0003 & & \\
\hline 3 & $0.017 \pm 75 \%$ & $0.094 \pm 29 \%$ & $0.129 \pm 18 \%$ & $0.0005 \pm 150 \%$ & $0.012 \pm 26 \%$ & $0.028 \pm 9 \%$ & 0.0003 & & \\
\hline 4 & $0.011 \pm 74 \%$ & $0.081 \pm 35 \%$ & $0.085 \pm 62 \%$ & $0.0006 \pm 36 \%$ & $0.013 \pm 33 \%$ & $0.031 \pm 14 \%$ & 0.0003 & 0.29 & 11 \\
\hline 5 & $0.009 \pm 54 \%$ & $0.041 \pm 27 \%$ & $0.062 \pm 48 \%$ & $0.0008 \pm 28 \%$ & $0.013 \pm 30 \%$ & $0.033 \pm 12 \%$ & 0.0003 & 0.21 & 10 \\
\hline
\end{tabular}

- Plasma mevalonic acid turnover, $\mathrm{L}(23,23)$, was fixed at 1.0 per $\min$ in all subjects. $\mathrm{L}(12,1)$ was fixed at 0.02 per $\mathrm{min}(4)$.

$\downarrow \mathrm{L}(\mathbf{4}, \mathbf{2 2})=\mathrm{L}(\mathbf{4}, \mathbf{2 1})$.

$\$ \mathrm{~L}(6,21)$ was fixed at 0.0003 per min based on published estimates for the normal FCR of LDL Apo B (18, 19). In subject 1 with a complete bile fistula, $\mathrm{L}(6,21)$ was fixed at 0.0006 per $\mathrm{min}$ in accord with recent reports of a twofold increase in LDL Apo B FCR in subjects taking cholestyramine (32), medication with physiologic effects similar to those of a bile fistula.

"Duration of the delay in conversion of plasma $\left[{ }^{14} \mathrm{C}\right]$ mevalonic acid, $\mathrm{C}(23)$, to NSC, $\mathrm{C}(1)$.

I Parameter value \pm FSD. 
TABLE V

Pool Sizes Derived from Compartmental Analysis

\begin{tabular}{ccrccc}
\hline Subject & $M(1)$ & $M(4)^{\bullet}$ & $M(6)$ & $M(12)$ & $M(21)+M(22) \bullet^{\prime}$ \\
\hline \multicolumn{5}{c}{$\mu$ mol } \\
& & \multicolumn{5}{c}{} \\
1 & 275 & 506 & 26,956 & 4,992 & 2,048 \\
2 & 105 & 649 & 15,043 & 6,901 & 2,441 \\
3 & 105 & 668 & 23,863 & 5,345 & 3,042 \\
4 & 105 & 1,034 & 20,000 & 6,327 & 2,836 \\
5 & 105 & 1,463 & 17,148 & 4,491 & 2,749 \\
\hline
\end{tabular}

- Calculated from the plasma concentration and plasma volume as $4.5 \%$ of kilograms body weight. The calculated values were then used to constrain the $\alpha$ - and $\beta$-free cholesterol compartment sizes in the kinetic analysis.

t $\mathrm{M}(21)$ plus $\mathrm{M}(22)$ represents the total $\beta$-FC pool. In the analysis 1:3 was arbitrarily proportioned as VLDL $[\mathrm{M}(22)]$ and $2: 3$ as LDL [M(21)].

assortment of labeled preparations administered, including $\left[{ }^{14} \mathrm{C}\right]$ mevalonic acid.

\section{DISCUSSION}

The data of the present report have provided some new information on several aspects of lipoprotein free cholesterol metabolism in man. It is quite clear that there are kinetic differences in the behavior of the FC in the $\alpha$ - and $\beta$-lipoproteins. The observed differences between the specific activities of free cholesterol in the $\alpha(\mathrm{HDL})$ and $\beta(\mathrm{VLDL}+\mathrm{LDL})$ lipoproteins were present after rapid separation of the lipoproteins by precipitation from plasma within $10 \mathrm{~min}$ of venipuncture, but not after 24-72 $\mathrm{h}$ of isolation from plasma in the ultracentrifuge (Table II). Extensive ex vivo exchange apparently occurs during ultracentrifugation. After the administration of $\left[{ }^{14} \mathrm{C}\right]$ mevalonic acid, $\alpha$-lipoprotein FC specific activity remained higher than the $\beta$-lipoprotein FC for $\sim 3 \mathrm{~h}$ (Figs. 4 and 5). Conversely, the specific activity of $\alpha$-lipoprotein FC remained considerably lower than the $\beta$-FC for $3 \mathrm{~h}$ after LDL- and VLDL-labeled free cholesterol were administered (Figs. 1, top, and 2). The multicompartmental model shown in Fig. 6 fit the data obtained following the administration of all labeled preparations. Modeling revealed that the FC in HDL directly exchanges with FC in VLDL, LDL, and tissues including a tissue that is enriched with newly synthesized cholesterol. In these in vivo studies, LDL and VLDL-FC exchanged with HDL and not with tissues. Within $20 \mathrm{~min}$, half the HDL-free cholesterol pool had exchanged with tissues. The metabolic functions of this unique property of HDL-FC are under investigation; one function probably involves exchange of free cholesterol between HDL and the hepatic bile acid and biliary cholesterol precursor pools. Also, the FC tissue exchange with HDL could potentially explain the protective effect of HDL against coronary artery disease.

The simulation analysis showed that almost all $\beta$ free cholesterol was transferred to HDL from where it could leave the plasma or cycle back to the $\beta$-fraction. The transport rate of LDL-free cholesterol to tissue via the Apo B pathway $(0.5-0.8 \mu \mathrm{mol} / \mathrm{min})$ was miniscule compared with the rate of $F C$ transport from LDL to HDL (47-90 $\mu \mathrm{mol} / \mathrm{min}$ ). Interestingly, the free cholesterol in both Apo B-rich lipoproteins, VLDL and LDL, appeared in a similar fashion in HDL (Fig. 2). The corresponding fractional rate constants, $L(4$, 21) and $L(4,22)$, were identical. Preliminary in vivo studies (unpublished observations) have shown that VLDL-FC and LDL-FC also appear (probably via HDL) in a similar fashion in erythrocytes, bile acids, and biliary cholesterol, supporting the conclusion that LDL- and VLDL-free cholesterol are metabolically and kinetically homogeneous. However, homogeneity of FC in these Apo B-rich particles cannot be proven until a method is available for the rapid isolation of VLDL from blood after venipuncture. The availability of such a technique could also help determine if plasma VLDL contains significant quantities of newly synthesized free cholesterol, which may be secreted with nascent VLDL from the liver and/or intestine.

When LDL labeled with FC was incubated in vitro with erythrocytes, in the absence of other blood components complete exchange of FC was observed (22, 23). Results of the present study suggested that, in vivo, LDL-FC and VLDL-FC do not exchange directly with tissues such as erythrocytes. This apparent discrepancy is probably explained by the absence of HDL from the in vitro studies; HDL, or a component thereof, may block the direct exchange of FC between LDL and membranes. This conclusion was supported by the report of Quarfordt and Hilderman (23) who showed that erythrocyte cholesterol exchanged faster with HDL than with LDL-FC when HDL, LDL, and erythrocytes were incubated together. These investigators did not discuss the possibility that LDL-FC exchanged indirectly with the erythrocytes, via HDL-FC as an intermediate, in the latter experiment.

To simulate the $\alpha$ - and $\beta$-free cholesterol specific activity curves it was necessary to invoke the presence of a hypothetical pool, C(12), which exchanged cholesterol extensively with HDL. The size of compartment 12 was constant among the subjects, average $5,600 \mu \mathrm{mol}$, and its fractional rate constant averaged $0.01 \mathrm{~min}^{-1}$ corresponding to a turnover time of about $1.5 \mathrm{~h}$. Although its identity is not known, indirect evidence suggests that neither plasma esterified cholesterol nor erythrocyte free cholesterol are part of C(12). The plasma esterified cholesterol pool sizes in the sub- 
jects in this study were $\sim 12,000 \mu \mathrm{m}$ (calculated from Table I, assuming plasma volume is $4.5 \%$ of body weight in kilograms). Also, the turnover time of plasma cholesterol esters is $\sim 36 \mathrm{~h}$ (24). Erythrocyte free cholesterol could not form part of $\mathrm{C}(12)$ because it is known to behave as a homogeneous FC pool that has a turnover time of $8 \mathrm{~h}(22,23,25)$, four-five times longer than the turnover time of cholesterol in $C(12)$. It is likely that a portion of compartment 12 is located in the liver and is comprised of the bile acid and biliary cholesterol precursor pools. These precursor pools are known to contain $\sim 500 \mu \mathrm{m}$ FC (minimum estimate), receive NSC directly, exchange preferentially with HDL-FC, and have turnover times of 1-1.5 h (4-7). All of these kinetic features are compatible with those of $\mathrm{C}(12)$. The studies of Gould et al. (26), using $\left[{ }^{14} \mathrm{C}\right]$ acetate in man to label cholesterol also suggested the presence of a hepatic cholesterol pool (in exchange with plasma FC) with a turnover time of $\sim 1.5 \mathrm{~h}(26)$. In summary, the data supports the conclusion that $\mathrm{C}(12)$ is extravascular cholesterol, part of which is in the liver.

Compartment 6, a less rapidly miscible pool of cholesterol compared with the others in this model, was necessary to simulate the plasma FC specific activities after $9 \mathrm{~h}$ and up to $3 \mathrm{~d}$ after the injection of labeled compounds. The approximate size and turnover time of $\mathrm{C}(6)$ was $20,000 \mu \mathrm{m}$ and $27 \mathrm{~h}$, respectively, suggesting that it could contain several metabolic pools including plasma esters and possibly erythrocyte cholesterol.

Undoubtably other small and large pools of cholesterol exist that are not shown in Fig. 6, such as pools 2 and 3 of Goodman et al. (27). However, preliminary simulation analysis has shown that the magnitude of cholesterol flux to and from these pools and plasma would be small relative to the fluxes between pools shown in Fig. 6. Pools 2 and 3 of Goodman et al. (27), had no appreciable effect on the decay curves for several days (or weeks) after injection of the labeled compounds.

The nature of the process that mediates the exchange of free cholesterol between HDL and tissues is open to speculation. Recent in vitro studies have shown that HDL can promote free cholesterol efflux from tissues (28-30). HDL is heterogeneous in this regard; $\mathrm{HDL}_{3}$ and the plasma fraction of density $>1.21$ (VHDL), both of which are enriched with Apo A-I, are the components of plasma that are potent acceptors of tissue free cholesterol (29). In our study we found no kinetic evidence for heterogeneity of the free cholesterol in HDL. These observations can be unified assuming that the specific acceptor "particle" mediates FC removal from tissue membranes; after appearing in plasma, FC on the acceptor becomes ho- mogeneous with FC in the entire HDL class of particles. Alternatively, HDL-FC may be metabolically heterogeneous and the FC exchange between HDL subclasses could occur at a rate too rapid to be detected by the present kinetic analysis and experimental design. It is also possible that the acceptor particle is not involved in the bidirectional exchange of FC between HDL and tissues, which was observed in our studies. Mechanisms for FC exchange and transport may exist in addition to the proposed acceptor particle. However, in light of current knowledge it seems reasonable to postulate that the acceptor particle does mediate the in vivo tissue efflux measured in the present study. There could be another class of particles that mediates the reverse process (HDL-FC to tissue FC), or the acceptor particle could mediate both limbs of the exchange process. Most likely, FC is constantly exchanging between HDL and tissue membranes; this exchange is mediated by the acceptor particle(s), and large net differences between efflux and influx can be detected only after a pertubation such as that induced in vitro by loading macrophages with cholesterol (30) or by loading HDL with FC (31).

\section{ACKNOWLEDGMENTS}

This research was supported in part by the National Institutes of Health grants AM 25920, AM 14668, and AM 23028, and the Veterans Administration.

\section{REFERENCES}

1. Goodman, D. S., and R. P. Noble. 1970. Cholesteryl ester turnover in human plasma lipoproteins during cholestyramine and clofibrate therapy. J. Lipid Res. 11: 183189.

2. Barter, P. J. 1974. Origin of esterified cholesterol transported in the very low density lipoproteins of human plasma. J. Lipid Res. 15: 11-19.

3. Glomset, J. A., E. T. Janssen, R. Kennedy, and J. Dobbins. 1966. Role of plasma lecithin: cholesterol acyltransferase in the metabolism of high density lipoproteins. $J$. Lipid Res. 7: 639-648.

4. Schwartz, C. C., M. Berman, Z. R. Vlahcevic, L. G. Halloran, D. H. Gregory, and L. Swell. 1978. Multicompartmental analysis of cholesterol metabolism in man. I. Characterization of the hepatic bile acid and biliary cholesterol precursor sites. J. Clin. Invest. 61: 408-423.

5. Schwartz, C. C., L. G. Halloran, Z. R. Vlahcevic, D. H. Gregory, and L. Swell. 1978. High and low density lipoprotein metabolism in man: preferential utilization of HDL free cholesterol by the liver for biliary cholesterol secretion. Science (Wash., DC). 200: 62-64.

6. Halloran, L. G., C. C. Schwartz, Z. R. Vlahcevic, R. M. Nisman, and L. Swell. 1978. Evidence for high-density lipoprotein-free cholesterol as the primary precursor for bile-acid synthesis in man. Surgery (St. Louis). 84: 1-7.

7. Portman, O. W., M. Alexander, and J. P. O'Malley. 1980. Metabolism of free and esterified cholesterol and apolipoproteins of plasma low and high density lipoproteins. Biochim. Biophys. Acta. 619: 545-558. 
8. Akanuma, Y., and J. Glomset. 1968. In vitro incorporation of cholesterol- ${ }^{14} \mathrm{C}$ into very low density lipoprotein cholesteryl esters. J. Lipid Res. 9: 620-629.

9. Gordon, T., W. P. Castelli, M. C. Hjortland, W. B. Kannel, and T. R. Dawber. 1977. High density lipoprotein as a protective factor against coronary heart disease. Am. J. Med. 62: 707.

10. Sperry, W., and M. Webb. 1950. Revision of Schoenheimer-Sperry method for cholesterol determination. $J$. Biol. Chem. 187: 97-106.

11. Hirsch, J., and E. H. Ahrens, Jr. 1958. The separation of complex lipide mixtures by the use of silicic acid chromatography. J. Biol. Chem. 233: 311-320.

12. Schwartz, C. C., Z. R. Vlahcevic, L. G. Halloran, and L. Swell. 1981. An in vivo evaluation in man of the transfer of esterified cholesterol between lipoproteins and into the liver and bile. Biochim. Biophys. Acta. 663: 143162.

13. Burstein, M., and H. R. Scholnick. 1973. Lipoproteinpolyanionic-metal interactions. In: Advances in Lipid Research. R. Paoletti and D. Kritchevsky, editors. Academic Press, Inc., New York. 11: 67-108.

14. Noble, R. P. 1968. Electrophoretic separation of plasma lipoproteins in agarose gel. J. Lipid Res. 9: 693-700.

15. Folch, J., M. Lees, and D. H. Sloan-Stanley. 1957. A simple method for the isolation and purification of total lipides from animal tissues. J. Biol. Chem. 226: 497-509.

16. Berman, M., and M. F. Weiss. 1974. SAAM Manual (revised) U. S. Department of Health, Education and Welfare, Public Health Service, Government Printing Office, Washington, DC.

17. Brown, M. S., and J. L. Goldstein. 1979. Receptor-mediated endocytosis: Insights from the lipoprotein receptor system. Proc. Natl. Acad. Sci. USA 76: 3330-3337.

18. Berman, M., M. Hall, R. I. Levy, S. Eisenberg, D. W. Bilheimer, R. D. Phair, and R. H. Goebel. 1978. Metabolism of apoB and apoC lipoproteins in man: kinetic studies in normal and hyperlipoproteinemic subjects. $J$. Lipid Res. 19: 38-56.

19. G. Sigurdsson, A. Nicoll, and B. Lewis. 1976. The metabolism of low density lipoprotein in endogeneous hypertriglyceridaemia. Eur. J. Clin. Invest. 6: 151-158.

20. Kekki, M., T. A. Miettinen, and B. Wahlstrom. 1977. Measurement of cholesterol synthesis in kinetically defined pools using fecal steroid analysis and double labeling technique in man. J. Lipid Res. 18: 99-114.
21. Grundy, S. M., and E. H. Ahrens. 1969. Measurements of cholesterol turnover, synthesis and absorption in man, carried out by isotope kinetic and sterol balance methods. J. Lipid Res. 10: 91-107.

22. Basford, J. M., J. Glover, and C. Green. 1964. Exchange of cholesterol between human $\beta$-lipoproteins and erythrocytes. Biochim. Biophys. Acta. 84: 764-766.

23. Quarfordt, S. H., and H. L. Hilderman. 1970. Quantitation of the in vitro free cholesterol exchange of human red cells and lipoproteins. J. Lipid Res. 11: 528-535.

24. Nestel, P. J., and E. A. Monger. 1967. Turnover of plasma esterified cholesterol in normocholesterolemic and hypercholesterolemia subjects and its relation to body build. J. Clin. Invest. 46: 967-974.

25. Lange, Y., J. Dolde, and T. L. Steck. 1981. The rate of transmembrane movement of cholesterol in the human erythrocyte. J. Biol. Chem. 256: 5321-5323.

26. Gould, R. G., G. V. LeRoy, G. T. Okita, J. J. Kabara, P. Keegan, and D. M. Bergenstal. The use of ${ }^{14} \mathrm{C}$-labeled acetate to study cholesterol metabolism in man. J. Lab. Clin. Med. 46: 372-384.

27. Goodman, D. S., R. P. Noble, and R. B. Dell. 1973. Three-pool model of the long-term turnover of plasma cholesterol in man. J. Lipid Res. 14: 178-188.

28. Stein, Y., M. C. Glangeaud, M. Fainaru, and O. Stein. 1975. The removal of cholesterol from aortic smooth muscle cells in culture and Landschutz ascites cells by fractions of human high-density apolipoprotein. Biochim. Biophys. Acta. 380: 106-118.

29. Oram, J. F., J. J. Albers, M. C. Cheung, and E. L. Bierman. 1981. The effects of subfractions of high density lipoprotein on cholesterol efflux from cultured fibroblasts. J. Biol. Chem. 256: 8348-8356.

30. Ho, Y. K., M. S. Brown, and J. L. Goldstein. 1980. Hydrolysis and excretion of cytoplasmic cholesteryl esters by macrophages: stimulation by high density lipoprotein and other agents. J. Lipid Res.. 21: 391-398.

31. Rothblat, G. H., L. Y. Arbogast, and E. K. Ray. 1978. Stimulation of esterified cholesterol accumulation in tissue culture cells exposed to high density lipoproteins enriched in free cholesterol. J. Lipid Res. 19: 350-358.

32. Shepherd, J., C. J. Packard, S. Bicker, T. D. Veitch Lawrie, and H. G. Morgan. 1980. Cholestyramine promotes receptor-mediated-low density-lipoprotein catabolism. N. Engl. J. Med. 302: 1219-1222. 